\title{
Improved Successive Cancellation Flip Decoding of Polar Codes Based on Error Distribution
}

\author{
Carlo Condo, Furkan Ercan, Warren J. Gross \\ Department of Electrical and Computer Engineering, McGill University, Montréal, Québec, Canada \\ Email: carlo.condo@mcgill.ca, furkan.ercan@mail.mcgill.ca,warren.gross@mcgill.ca
}

\begin{abstract}
Polar codes are a class of linear block codes that provably achieves channel capacity, and have been selected as a coding scheme for $5^{\text {th }}$ generation wireless communication standards. Successive-cancellation (SC) decoding of polar codes has mediocre error-correction performance on short to moderate codeword lengths: the SC-Flip decoding algorithm is one of the solutions that have been proposed to overcome this issue. On the other hand, SC-Flip has a higher implementation complexity compared to SC due to the required log-likelihood ratio (LLR) selection and sorting process. Moreover, it requires a high number of iterations to reach good error-correction performance. In this work, we propose two techniques to improve the SC-Flip decoding algorithm for low-rate codes, based on the observation of channel-induced error distributions. The first one is a fixed index selection (FIS) scheme to avoid the substantial implementation cost of LLR selection and sorting with no cost on error-correction performance. The second is an enhanced index selection (EIS) criterion to improve the error-correction performance of SCFlip decoding. A reduction of $24.6 \%$ in the implementation cost of logic elements is estimated with the FIS approach, while simulation results show that EIS leads to an improvement on error-correction performance improvement up to $0.42 \mathrm{~dB}$ at a target FER of $10^{-4}$.
\end{abstract}

\section{INTRODUCTION}

Polar codes [1] are error-correcting codes proven to achieve channel capacity with infinite code length. They have been selected as a coding scheme in the $5^{\text {th }}$ generation wireless systems standards $(5 \mathrm{G})$, within the enhanced mobile broadband communication scenario (eMBB), and are being considered for both ultra-reliable low-latency communications (URLLC) and massive machine-type communications (mMTC) [2]. The variety of system parameters foreseen in 5G demand improved decoding algorithms with a focus on reliable communications and low power consumption.

In [1], the successive-cancellation (SC) decoding of polar codes was proposed. It is able to achieve channel capacity when the code length tends to infinite, but its error-correction performance degrades at shorter lengths. To overcome this limitation, the SC-List decoding algorithm was introduced in [3]. The improved error-correction performance comes at the cost of longer latency and higher complexity: various contributions have improved speed and reduced implementation cost [4]-[6].

\footnotetext{
${ }^{0}$ This version of the manuscript corrects an error in the previous ArXiv version, as well as the published version in IEEE Xplore under the same title, which has the DOI:10.1109/WCNCW.2018.8368991. The corrections include all the simulations of SC-Flip-based and SC-Oracle decoders, along with associated comments in-text.
}

Successive-cancellation flip (SC-Flip) decoding [7] takes a different approach to the improvement of SC, relying on an implementation complexity lower than that of SC-List, mainly sacrificing average decoding latency, and providing error-correction performance comparable to that of SC-List with a list size of 2. SC-Flip relies on multiple subsequent applications of SC, and on the identification of the most likely points in which the SC algorithm made a wrong decision. This operation is based on the magnitude of logarithmic likelihood ratios (LLRs) associated to the estimated codeword bits, and it is costly to implement. Modifications proposed in [8], [9] have shown that the error identification and correction process of SC-Flip can be greatly improved. A decoding approach similar to [5] has been recently proposed for SC-Flip in [10], reducing latency at no cost in error-correction performance, but substantially increasing the implementation complexity of the decoder.

In this work, we propose two techniques to improve SCFlip decoding, based on the distribution of the first wrong estimation incurred by SC. The first method simplifies the wrong estimation identification process without degrading the error-correction performance, leading to substantial complexity reduction, while the second method uses the error distribution to restrict the search space to the bit indices most likely to incur errors. Simulation results show the effectiveness of the proposed techniques for low-rate polar codes, over a wide range frame error rate (FER) intervals.

The remainder of this work is organized as follows: in Section II polar codes and their decoding algorithms are introduced. In Section III the proposed techniques are detailed, and simulation results, together with comparison with the state of the art, are presented in Section IV] Conclusions are drawn in Section $\mathrm{V}$.

\section{PRELIMINARIES}

\section{A. Polar Codes}

A polar code $P C(N, K)$ of code length $N$ and rate $R=$ $K / N$ is a linear block code that divides $N=2^{n}, n \in \mathbb{Z}^{+}$ bit-channels in $K$ reliable ones and $N-K$ unreliable ones. Information bits are transmitted through the reliable channels, whereas unreliable channels are fixed to a value known by both transmitter and receiver, and are thus called frozen bits.

Polar codes are encoded through the following matrix multiplication:

$$
x_{0}^{N-1}=u_{0}^{N-1} G^{\otimes n},
$$




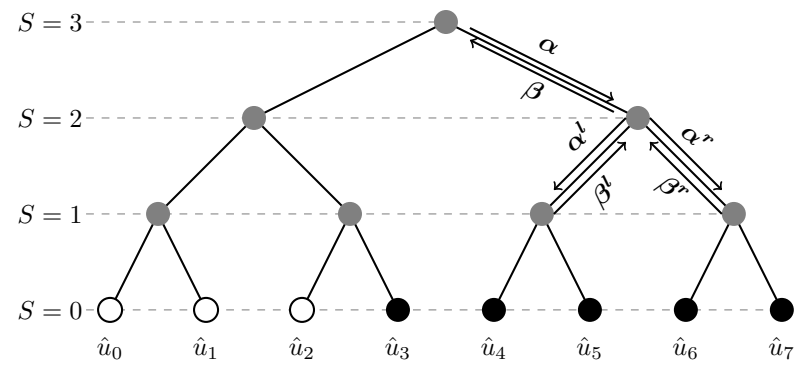

Fig. 1. Successive-cancellation decoding tree for a $P C(8,5)$ code.

where $\boldsymbol{x}_{\mathbf{0}}^{\boldsymbol{N}-\mathbf{1}}=\left\{x_{0}, x_{1}, \ldots, x_{N-1}\right\}$ represents the encoded vector, $\boldsymbol{u}_{\mathbf{0}}^{N-1}=\left\{u_{0}, u_{1}, \ldots, u_{N-1}\right\}$ is the input vector, and the generator matrix $G^{\otimes n}$ is obtained as the $n$-th Kronecker product of the polarization matrix $G=\left[\begin{array}{ll}1 & 0 \\ 1 & 1\end{array}\right]$. Due to the recursive nature of the encoding process, an $N$-length polar code can be interpreted as the concatenation of two polar codes of length $N / 2$.

The scheduling of operations required by the SC decoding algorithm allows to see its process as a binary tree search, where the tree is explored depth-first, with priority given to the left branch. Fig. 1 portrays an example of SC decoding tree, for $P C(8,5)$. Each node receives from its parent a vector of LLRs $\boldsymbol{\alpha}=\left\{\alpha_{0}, \alpha_{1}, \ldots, \alpha_{2^{S}-1}\right\}$. Each node at stage $S$ computes the left $\boldsymbol{\alpha}^{\boldsymbol{l}}=\left\{\alpha_{0}^{l}, \alpha_{1}^{l}, \ldots, \alpha_{2^{S-1}-1}^{l}\right\}$ and right $\boldsymbol{\alpha}^{\boldsymbol{r}}=$ $\left\{\alpha_{0}^{r}, \alpha_{1}^{r}, \ldots, \alpha_{2 S-1-1}^{r}\right\}$ LLR vectors sent to child nodes as

$$
\begin{aligned}
& \alpha_{i}^{l}=\operatorname{sgn}\left(\alpha_{i}\right) \operatorname{sgn}\left(\alpha_{i+2^{S-1}}\right) \min \left(\left|\alpha_{i}\right|,\left|\alpha_{i+2^{S-1}}\right|\right), \\
& \alpha_{i}^{r}=\alpha_{i+2^{S-1}}+\left(1-2 \beta_{i}^{l}\right) \alpha_{i} .
\end{aligned}
$$

The LLRs at the root node are initialized as the channel LLR $\boldsymbol{y}_{0}^{N-1}$. Nodes receive the partial sums $\boldsymbol{\beta}$ from their left $\boldsymbol{\beta}^{\boldsymbol{l}}=$ $\left\{\beta_{0}^{l}, \beta_{1}^{l}, \ldots, \beta_{2^{S-1}-1}^{l}\right\}$ and right $\boldsymbol{\beta}^{r}=\left\{\beta_{0}^{r}, \beta_{1}^{r}, \ldots, \beta_{2^{S-1}-1}^{r}\right\}$ child node:

$$
\beta_{i}= \begin{cases}\beta_{i}^{l} \oplus \beta_{i}^{r}, & \text { if } i \leq 2^{S-1} \\ \beta_{i}^{r}, & \text { otherwise. }\end{cases}
$$

where $\oplus$ is the bitwise XOR operation, and $0 \leq i<2^{S}$. At leaf nodes, the $\beta$ value and the estimated bit vector $\hat{\boldsymbol{u}}_{\mathbf{0}}^{N-1}$ are computed as

$$
\beta_{i}= \begin{cases}0, & \text { when } \alpha_{i} \geq 0 \text { or } i \in \Phi \\ 1, & \text { otherwise. }\end{cases}
$$

The SC-List decoding algorithm [3] improves the errorcorrection performance of SC by relying on $L$ parallel SC decoding paths. Each path is associated to a metric, that helps deciding which of the $L$ paths is more likely to be correct.

\section{B. Successive-Cancellation Flip Decoding}

To improve the error-correction performance of SC, in [7] the SC-Flip decoding algorithm was proposed. It is based on the observation that a failed SC decoding process is due to either an incorrect bit estimation caused by noise introduced by the channel or, due to the sequential nature of SC, to a previous incorrect bit estimation. Thus, the first wrong estimation is always caused by the channel noise. To evaluate the impact of wrong estimations, a genie-like decoder called SC-Oracle was created: using foreknowledge of the transmitted codeword, it identifies the channel-induced errors and ensures that SC estimates the bit correctly. SC-Oracle shows that avoiding all wrong decisions caused by the channel noise results in significantly improved SC error-correction performance. Moreover, simulations with SC-Oracle have shown that a failed SC decoding is mostly due to a single wrong decision caused by channel noise, an event that denote as $E_{1}$.

The SC-Flip algorithm attempts the identification and correction of $E_{1}$ events. A cyclic redundancy check (CRC) code with a $C$-bit remainder is used to encode the information bits. If the CRC check passes at the end of SC decoding, the estimated codeword is assumed correct. In case the CRC check detects an error, the $T_{\max }$ LLRs with the smallest magnitude, representing the bit estimations with lowest reliability, are stored and sorted. The bit associated to the smallest LLR is flipped, and SC is is applied to the part of the decoding tree that follows it. This process is repeated for all the $T_{\max }$ indices, or until the CRC passes.

In general, increasing $T_{\max }$ improves the error-correction performance of SC-Flip, bringing it ever closer to that of SCOracle. In fact, the performance gap between SC-Flip and SC-Oracle is due to two possible cases: either the estimated codeword with a correct CRC check still contains errors, or the decoding stopped after reaching the maximum number of iterations $T_{\max }$ without being successful.

Further improvements for SC-Flip decoding algorithm have been recently proposed in [8] and [9], where a generalized SC-Flip algorithm uses nested flips to correct more than one erroneous decision with a single CRC, with a simulationbased scaling metric also introduced to help the baseline SCFlip decoder detect the erroneous bit indices more accurately. Simulation results show an improvement of $0.4 \mathrm{~dB}$ when $T_{\max }=10$ with respect to SC-Flip. The implementation of this method requires parallel decoders similar to SC-List.

\section{ImProved SucCessive-CANCELLATION FliP DECODING}

We propose two new techniques to improve the errorcorrection performance of SC-flip decoding, and to reduce the implementation complexity of SC-Flip decoders.

\section{A. Fixed Index Selection}

In [7], it was shown that the average number of iterations for SC-Flip decoding converges to that of a single SC decoder at moderate to high $E_{b} / N_{0}$. However, a single iteration of SCFlip requires additional operations with respect to SC. The standard SC-Flip decoding algorithm decides which bits to flip by identifying the $T_{\max }$ least reliable bit estimations among the non-frozen indices. Since the estimation reliability is directly associated to the magnitude of its LLR value (5), they correspond to the $T_{\max }$ LLRs with the smallest magnitude. The associated bits are flipped in ascending order starting from the one with she smallest $|\alpha|$ : thus, the construction of the 


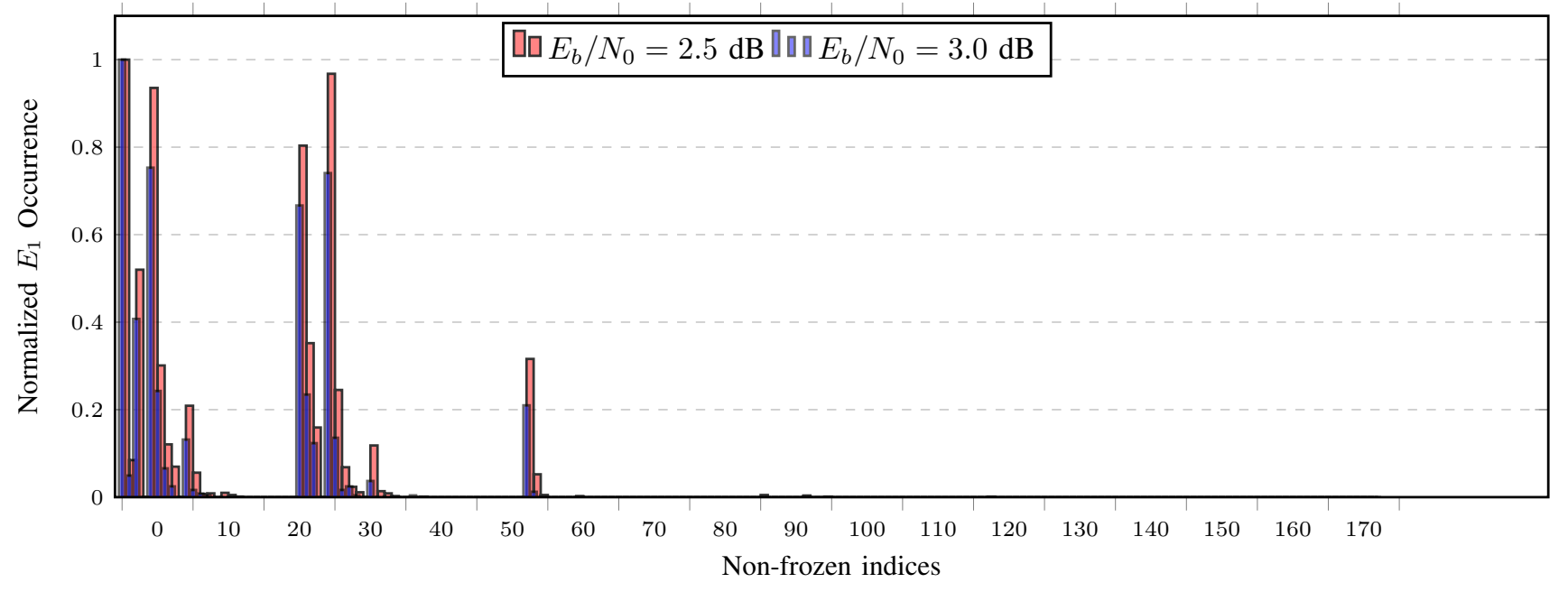

Fig. 2. Normalized distribution of $E_{1}$ occurrence for $P C(1024,170), C=8,5 \times 10^{5}$ simulated frames.

list requires the $T_{\max }$ LLRs to be not only selected, but also sorted. This operation results in substantial implementation cost, that is the main contributor to the additional hardware complexity of SC-Flip decoders with respect to SC decoders.

In Fig. 2 it is shown the normalized distribution of $E_{1}$ occurrence over the non-frozen bit indices for $P C(1024,170)$, with $C=8$, obtained simulating $5 \times 10^{5}$ frames, for two $E_{b} / N_{0}$ points. It can be noticed that bits have different probability of $E_{1}$, with a select few having a higher probability of $E_{1}$ than the remaining ones, and the majority of them not incurring any error. The height and position of the error distribution spikes can be used to determine, off-line, which bits to flip and in which order. In the provided example for $E_{b} / N_{0}=3.0 \mathrm{~dB}$, considering the non-frozen bits indexed from 0 to $K+C-1$, the first flipped bit would be that at index 0 , corresponding to the highest $E_{1}$ occurrence, followed by those at index 29, 4,25 , and so on, in descending probability of $E_{1}$ occurrence order, until $T_{\max }$ attempts have been reached or the CRC check passes. Determining which bits to flip off-line allows to avoid the LLR selection and sorting logic. Thus, the resulting SC-Flip decoder has an implementation cost negligibly different from that of an SC decoder.

\section{B. Enhanced Index Selection}

It is possible to use the error distribution information to increase the effectiveness of the standard LLR-based bit flipping selection scheme of SC-Flip. In its original formulation [7], SC-Flip chooses which $T_{\max }$ bits to flip among all the $K+C$ non-frozen bits in the code. However, from Fig. 2 we can see that the majority of information bits have very low error probability, that can be considered negligible depending on the target FER. Consequently, it is possible to restrict the LLR selection to the set of bit indices corresponding to high enough probability of $E_{1}$.

This can improve the error-correction performance by increasing the chance of the wrong estimation to be included within a limited number of $T_{\max }$ indices, automatically ex-

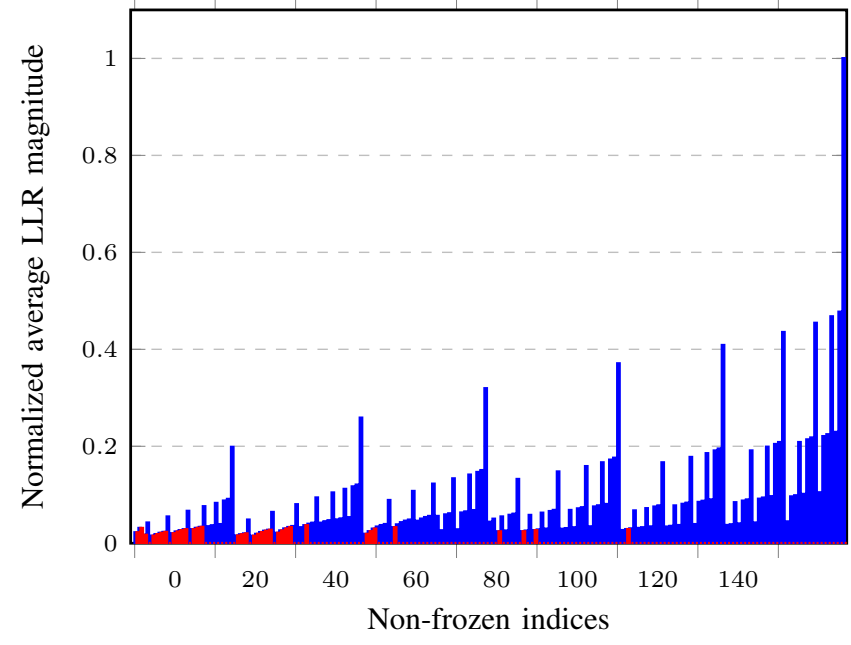

Fig. 3. Normalized average LLR magnitude at each non-frozen bit index for $P C(1024,170)$ at $E_{b} / N_{0}=2.5 \mathrm{~dB}$ and $C=8$. Indices highlighted in red correspond to the non-zero occurrence of $E_{1}$ shown in Fig. 2

cluding LLRs that have an averagely low magnitude, but correspond to indices with low error probability. Let us in fact observe Fig. 3, that shows the average LLR magnitude for each information bit index for $P C(1024,170), C=8$, at $E_{b} / N_{0}=2.5 \mathrm{~dB}$. It can be seen that the average LLR magnitude $|\alpha|$ can vary quite a lot depending on the bit. Highlighted in red are the LLRs whose indices correspond to the non-zero occurrences of $E_{1}$ shown in Fig. 2. many LLRs of comparable or even smaller average magnitude are instead associated to indices with very low probability of $E_{1}$. These LLRs can feature among the $T_{\max }$ with the smallest absolute value and be selected by the standard SC-Flip algorithm, but correspond to the erroneous bit in a negligible number of cases. On the other hand, their being taken in account can force the actual wrong estimation out of the $T_{\max }$ flipped indices.

The error distribution as shown in Fig. 2 depends on the 


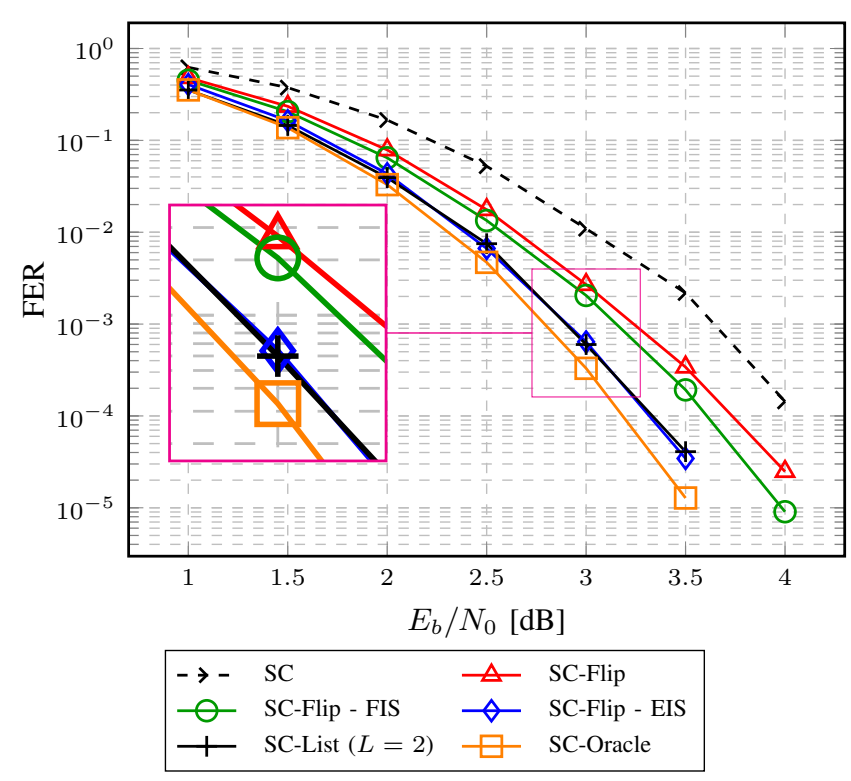

Fig. 4. FER curves for for $P C(1024,170), C=8, T_{\max }=10$ with different decoding approaches.

number of simulated frames we are considering. With enough frames, every information bit index is associated with a nonzero $E_{1}$ occurrence: the zero error probability in Fig. 2 means that no errors were encountered over the $5 \times 10^{5}$ simulated frames. Given a target FER, it is possible to exclude from the considered indices all those associated to error probabilities lower than a certain threshold, in order to maximize the chances of identifying the wrong estimation within $T_{\max }$ attempts. Moreover, to take in account the relative probability of $E_{1}$ occurrence, before sorting LLRs are scaled with respect to the the associated $E_{1}$ occurrence (see Fig. 2).

\section{Simulation Results}

The error-correction performance of the proposed methods has been evaluated and compared against the state of the art. Fig. 4 and Fig. 5 show the FER for $P C(1024,170)$ and $P C(1024,256)$ respectively, with $C=8$. Both codes have been constructed targeting a signal-to-noise ratio of $2.5 \mathrm{~dB}$, and simulations have been run on AWGN channel and BPSK modulation. Along with standard SC, curves are plot for SC-Flip [7], SC-List [3], SC-Oracle, and SC-Flip with the proposed fixed index selection (FIS) and enhanced index selection (EIS). The maximum number of iterations has been set to $T_{\max }=10$ for all flip-based algorithms. The SC-Oracle curves have been obtained considering $\mathrm{K}+\mathrm{C}$ non-frozen bits.

The FER of SC is the highest among all considered algorithms, since all incarnations of SC-Flip substantially improve on its error correction performance. One exception to this is SC-Flip with $P C(1024,256)$; its performance matches that of $\mathrm{SC}$, due to the degradation caused by errors on the additional non-frozen CRC bits. On the other hand, the SC-Oracle curve represents the case in which all $E_{1}$ occurrences are successfully corrected, and thus can be interpreted as the lower

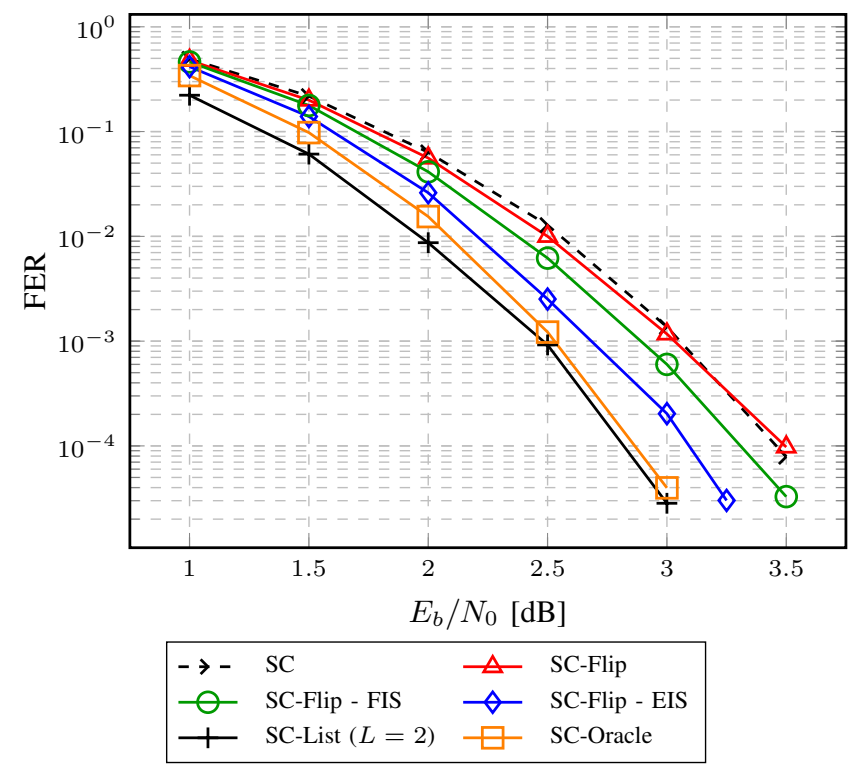

Fig. 5. FER curves $P C(1024,256), C=8, T_{\max }=10$ with different decoding approaches.

bound of flip-based FER. The SC-Flip decoding algorithm improves the error-correction performance of SC, but in both Fig. 4 and Fig. 5 a substantial gap from the SC-Oracle bound can be observed, consisting of $0.55 \mathrm{~dB}$ for $P C(1024,170)$ and of $0.63 \mathrm{~dB}$ for $P C(1024,256)$ at $\mathrm{FER}=10^{-4}$.

SC-Flip with FIS is shown to perform slightly better than standard SC-Flip for $P C(1024,170)$ in Fig. 4, with a gain of $0.12 \mathrm{~dB}$ at $\mathrm{FER}=10^{-4}$. In case of $P C(1024,256)$, the gain of the FIS criterion compared to baseline SC-Flip is $0.19 \mathrm{~dB}$. The $T_{\max }=10$ indices considered by the method are those corresponding to the highest probabilities of $E_{1}$. This technique allows to substantially reduce the implementation complexity of an SC-Flip decoder. The second column of Table I reports the number and type of operations required by a standard SC-Flip decoder, assuming a semi-parallel structure similar to [11], and omitting the memory requirements. The complexity is divided among the three main operations that an SC-Flip decoder has to perform: F (2), G (3), C (4), and the sorting and selection of the $T_{\max }$ indices to flip. Basic modules as comparators, XORs, multiplexers, adders and registers are used to approximate the implementation complexity; the number of parallel processing elements $P e$, along with the LLR quantization bits $Q$, also affect the total cost. It can be seen that FIS allows to completely avoid the logic cost of the sorter function. To get a sense of its impact on the total logic complexity, we consider the XOR block having a cost of 1 , the multiplexer a cost of 3 , the adder and the comparator a cost of 5 and the register a cost of 4 . Supposing an architecture with $P e=32, Q=6$ and $T_{\max }=10$, the sorter accounts for $24.6 \%$ of the total logic complexity.

Compared to SC-Flip, the EIS approach allows for $0.4 \mathrm{~dB}$ and $0.42 \mathrm{~dB}$ gain for $P C(1024,170)$ and $P C(1024,256)$ respectively. In Fig. 4 a total of 26 indices are considered, 
TABLE I

IMPLEMENTATION LOGIC COST BREAKDOWN FOR SC-FLIP AND SC-FLIP - FIS

\begin{tabular}{l|c|c}
\hline & SC-Flip & SC-Flip - FIS \\
\hline \multirow{5}{*}{$\mathrm{F}$} & $P_{e} \times Q \times$ Comparator & $P_{e} \times Q \times$ Comparator \\
& $2 \times P_{e} \times \mathrm{XOR}$ & $2 \times P_{e} \times \mathrm{XOR}$ \\
& $P_{e} \times Q \times \mathrm{MUX}$ & $P_{e} \times Q \times \mathrm{MUX}$ \\
\hline \multirow{2}{*}{$\mathrm{G}$} & $P_{e} \times Q \times \mathrm{Sum}$ & $P_{e} \times Q \times \mathrm{Sum}$ \\
& $P_{e} \times \mathrm{MUX}$ & $P_{e} \times \mathrm{MUX}$ \\
\hline $\mathrm{C}$ & $P_{e} \times \mathrm{MUX}$ & $P_{e} \times \mathrm{MUX}$ \\
\hline \multirow{3}{*}{ Sorter } & $T_{\max } \times Q \times \mathrm{D}-\mathrm{FF}$ & N/A \\
& $T_{\max } \times Q \times \mathrm{Comparator}$ & \\
& $2 \times T_{\max } \times Q \times \mathrm{MUX}$ &
\end{tabular}

while 45 are taken in account for the higher rate case portrayed in Fig. 5. The FER of SC-Flip with EIS matches to that of SC-List with $L=2$. In the case of $P C(1024,256)$, the errorcorrection performance of the list decoder outperforms SCOracle decoder. It was shown in [12] that while an outer CRC code improves the error-correction performance of SC-List decoding significantly at medium to high code rates, at low code rates the $\mathrm{CRC}$ is detrimental. Thus, for a fair comparison, we did not consider any CRC in the SC-List curves. The indices used by EIS have been found simulating a total of $1 \times 10^{6}$ frames, and selecting those with the higher probability of $E_{1}$. The impact of different number of considered indices in EIS is shown in Fig. 6 for $P C(1024,170)$ : it can be seen that decreasing it tends to degrade the error-correction performance, while increasing it does not bring substantial advantage. This remains true as long as the number of indices considered by EIS is substantially lower than $K+C$, at which point the FER will degrade to the same obtained by standard SC-Flip.

As the code rate increases, the error correction performance of both FIS and EIS starts degrading if $T_{\max }$ is not increased. This is due to the fact that as the number of information bits in the code rises, the distribution of $E_{1}$ occurrence spreads over more bit indices. This results in a higher number of indices that need to be considered by the proposed method: a higher $T_{\max }$ is thus needed to maintain the error-correction performance.

Fig. 7 depicts the average number of iterations for different decoding approaches, where an iteration is defined as an application of the SC algorithm over the $N$ codeword bits. It can be observed that at low $E_{b} / N_{0}$ points, SC-Flip with the FIS technique has up to $44 \%$ higher average iterations with respect to standard SC-Flip [7], while the use of EIS entails an increment of up to $30 \%$. Both EIS and FIS reduce the number of cases in which SC-Flip reaches the $T_{\max }$ attempts, lowering the average number of iterations. For example, the SC-Flip decoding algorithm with $P C(1024,170)$ at $E_{b} / N_{0}=1.0 \mathrm{~dB}$ is not able to find the correct codeword $45 \%$ of the time, with $T_{\max }=10$ and $C=8$. With the EIS criterion, this failure rate drops by $12 \%$. However, this positive contribution is offset by the position of the indices with high probability of $E_{1}$ occurrence, as shown in Fig. 2,3. The majority of these indices are in fact found towards the beginning of the

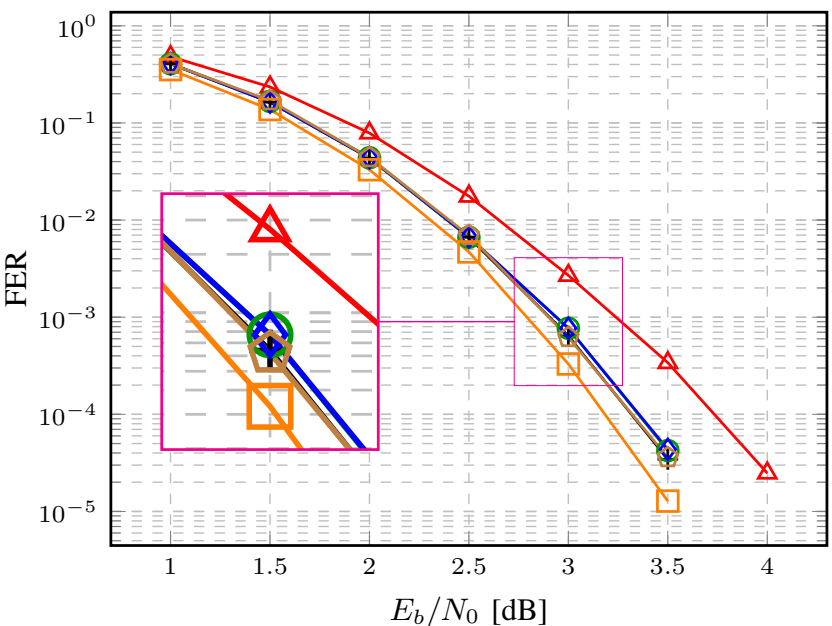

\begin{tabular}{|llll|}
\hline$\triangle$ & SC-Flip & $\longleftarrow$ & SC-Flip - EIS 36 indices \\
- & SC-Flip - EIS 26 indices & $\checkmark$ & SC-Flip - EIS 20 indices \\
$\triangle$ & SC-Flip - EIS 17 indices & $\square$ & SC-Oracle \\
\hline
\end{tabular}

Fig. 6. FER curves for $P C(1024,170), C=8, T_{\max }=10$ with different decoding approaches and number of indices considered by SC-Flip - EIS.

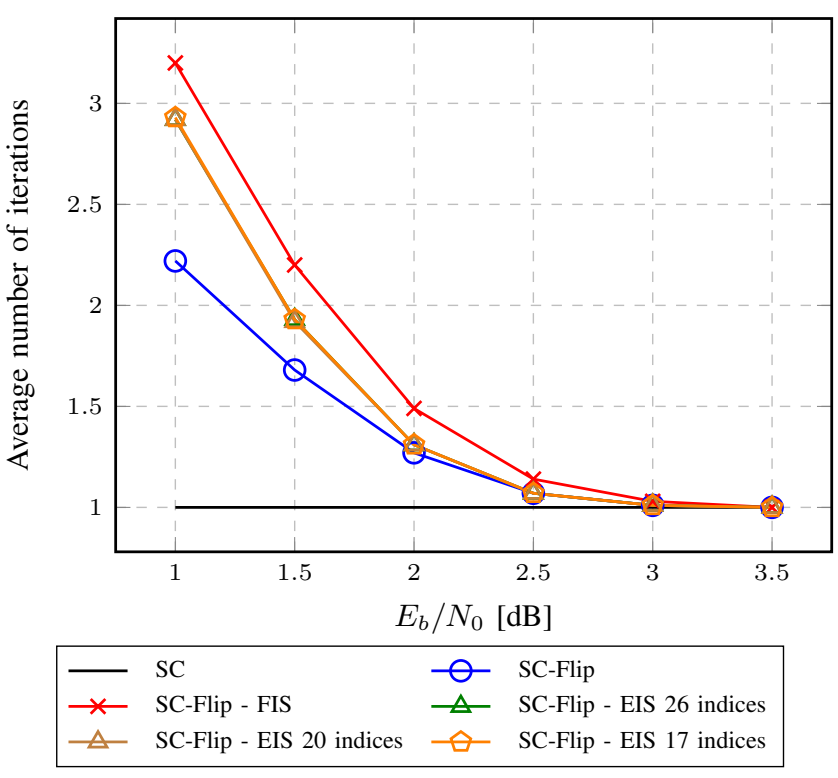

Fig. 7. Average number of iterations for $P C(1024,170), C=8, T_{\max }=$ 10 with different decoding approaches.

codeword: thus, each additional decoding attempt requires almost a full iteration. On the contrary, in standard SC-Flip there are no constraints on which indices to consider, and at low $E_{b} / N_{0}$, the channel noise causes most LLRs to have very low magnitude. Consequently, the $T_{\max }$ indices can be spread out over the whole codeword, leading to standard SCFlip having a lower average number of iterations. At higher $E_{b} / N_{0}$, where the FER is of interest, the average number of iterations quickly converges to 1 for all methods. 


\section{CONCLUSION}

In this work, we have proposed two techniques to reduce the implementation complexity and reduce the FER of the SC-Flip decoding algorithm. They are based on the identification of the bit indices where a channel-induced error is most likely to occur. The proposed techniques have shown significant improvement over SC-Flip when applied to lowrate polar codes. The fixed index selection method has shown an estimated $24.6 \%$ implementation complexity reduction at no cost in error-correction performance, while the enhanced index selection has been observed to bring up to $0.42 \mathrm{~dB}$ gain in FER.

\section{REFERENCES}

[1] E. Arıkan, "Channel polarization: A method for constructing capacityachieving codes for symmetric binary-input memoryless channels," IEEE Transactions on Information Theory, vol. 55, no. 7, pp. 3051-3073, July 2009.

[2] 3GPP, "Final report of 3GPP TSG RAN WG1 \#87 v1.0.0," http://www.3gpp.org/ftp/tsg_ran/WG1_RL1/TSGR1_87/Report/Final_ Minutes_report_RAN1\%2387_v100.zip Reno, USA, November 2016.

[3] I. Tal and A. Vardy, "List decoding of polar codes," IEEE Transactions on Information Theory, vol. 61, no. 5, pp. 2213-2226, May 2015.

[4] A. Balatsoukas-Stimming, M. B. Parizi, and A. Burg, "LLR-based successive cancellation list decoding of polar codes," IEEE Transactions on Signal Processing, vol. 63, no. 19, pp. 5165-5179, Oct 2015.

[5] S. A. Hashemi, C. Condo, and W. J. Gross, "Fast and flexible successivecancellation list decoders for polar codes," CoRR, vol. abs/1703.08208, 2017. [Online]. Available: http://arxiv.org/abs/1703.08208

[6] S. A. Hashemi, C. Condo, F. Ercan, and W. J. Gross, "Memory-efficient polar decoders," IEEE Journal on Emerging and Selected Topics in Circuits and Systems, vol. PP, no. 99, pp. 1-1, 2017.

[7] O. Afisiadis, A. Balatsoukas-Stimming, and A. Burg, "A low-complexity improved successive cancellation decoder for polar codes," in Asilomar Conference on Signals, Systems and Computers, Nov 2014, pp. 21162120.

[8] L. Chandesris, V. Savin, and D. Declercq, "An improved SCFlip decoder for polar codes," in IEEE Global Communications Conference (GLOBECOM), Dec 2016, pp. 1-6.

[9] L. Chandesris, V. Savin, and D. Declercq, "Dynamic-SCFlip decoding of polar codes," CoRR, vol. abs/1703.04414, 2017. [Online]. Available: http://arxiv.org/abs/1703.04414

[10] P. Giard and A. Burg, "Fast-SSC-Flip Decoding of Polar Codes," IEEE Wireless Communications and Networking Conference, 2018, to appear. [Online]. Available: http://arxiv.org/abs/1712.00256

[11] A. J. Raymond and W. J. Gross, "A scalable successive-cancellation decoder for polar codes," IEEE Transactions on Signal Processing, vol. 62, no. 20, pp. 5339-5347, Oct 2014.

[12] S. A. Hashemi, C. Condo, F. Ercan, and W. Gross, "On the performance of polar codes for 5G eMBB control channel," in Asilomar Conference on Signals, Systems and Computers, 2017, to appear. 